\title{
Fabrication of ZnO Hollow Microspheres Organized by ZnO Nanorods via Single-step Hydrothermal Process
}

\author{
Ying-Ying LI \\ Public Security Fire Forces College, Kunming 650208, China \\ waiwailyy@163.com \\ ${ }^{*}$ Corresponding author
}

Keywords: ZnO, Nanorods, Hydrothermal, Optical properties.

\begin{abstract}
Micrometer scale hollow $\mathrm{ZnO}$ dandelions organized by $\mathrm{ZnO}$ nanorods were prepared by single-step hydrothermal process. The diameter of the nanorods with the pencil shape tip is estimated to be $150-500 \mathrm{~nm}$. The length of the nanorods is more than $2 \mu \mathrm{m}$. Structure analysis indicated that the as-prepared products were wurtzite structure with high crystallinity. The $\mathrm{ZnO}$ nanorods microspheres were found to have excellent UV emission properties at room temperature. These kinds of hollow spherical structures may find potential applications in photocatalysis, light-weight composite fillers, UV nano/micro-optoemission devices and photoanodes of dye-sensitized solar cells.
\end{abstract}

\section{Introduction}

The Kirkendall effect normally refers to comparative diffusive migrations among different atomic species in metals and/or alloys under thermally activated conditions ${ }^{[1]}$. For example, because of the difference in atomic diffusivities, zinc diffuses into the copper faster than the copper diffuses into the brass in a brass-copper interface ${ }^{[2]}$. A common result from this process is generation of porosity in the low-melting component side of the diffusion couple. Recently, a process analogous to the Kirkendall effect has been successfully developed for nanoscale fabrication of a variety of hollow crystals, including core-shell metal-metal-oxide structures ${ }^{[3]}$.

As a special class of materials, metal oxide hollow spheres in nano/microscales have attracted great investigative interest due to their unique properties, such as low density, high specific surface area and good permeability, and so on. This kind of structure has wide potential applications in adsorption, catalysis, biomedical diagnosis, delivery of drugs, photonic crystals, acoustic insulation, and light-weight composite fillers ${ }^{[4-7]}$. The low dimensional, wide band gap $(3.37 \mathrm{eV})$ semiconductor-zinc oxide $(\mathrm{ZnO})$ with large exciton binding energy $(60 \mathrm{meV})$ at room temperature has been so far applied in many aspects, such as nanoscale lasers ${ }^{[8]}$, piezoelectric devices ${ }^{[9]}$, and sensors ${ }^{[10]}$. Especially, hollow $\mathrm{ZnO}$ spheres with nanowires grown on both inner and outer surfaces of the spherical shells are very beneficial to applications in photocatalytic, acoustic insulation, light-weight composite fillers, and photoanodes of dye-sensitized solar cells owing to their densities and much higher surface to volume ratio as compared to solid spheres and hollow shell structures without nanowires ${ }^{[11]}$.

In this article, micrometer scale hollow $\mathrm{ZnO}$ dandelions organized by $\mathrm{ZnO}$ nanorods were formed via hydrothermal method, following a modified Kirkendall process. To character the crystal structures of samples, the X-ray diffraction (XRD) were investigated. The surface morphology was studied with a scanning electron microscopy (SEM). The optical properties of the $\mathrm{ZnO}$ nanorods arrays were investigated by the photoluminescence (PL) spectrometer and optical absorption spectra. In principle, a lot of metal oxides and metal-semiconductor composites can be obtained in this synthetic architecture. Hopefully, they can be applied in many fields, such as light-generated electrons, three-dimensional lasing, and new photocatalysts. 


\section{Experimental}

\section{Materials}

All chemicals were analytical-grade reagents and were purchased from Shanghai Chemical Reagent Corp. The zinc powder (99.99\% purity) was polished, washed by acetone, ethanol and distilled water for several times, and dried in the oven. Tetramethylammoniumhydroxide (TMAOH, $25 \%$ ) were used as reactants without further purification.

\section{Preparation of Micrometer Scale Hollow ZnO Dandelions Organized by ZnO Nanorods}

$5 \mathrm{mmol}$ of the $\mathrm{Zn}$ powder were put into a $50 \mathrm{mo}$ Teflon-lined stainless autoclave within $35 \mathrm{ml}$ aqueous solution of $0.5 \mathrm{M}$ TMAOH. The autoclave was heated to $180^{\circ} \mathrm{C}$ for $2-24 \mathrm{~h}$ in the oven. After the reaction, the specimen was completely washed with absolute ethanol and Milli-Q water for several times. The sample was dried in vacuum at $60^{\circ} \mathrm{C}$ for $12 \mathrm{~h}$.

\section{Characterization}

The morphologies of the as-synthesized $\mathrm{ZnO}$ nanorods arrays were observed by field-emission scanning electron microscopy (FE-SEM, LEO1550). The crystal structures of the obtained samples were characterized by $\mathrm{X}$-ray diffraction with graphite monochromatized $\mathrm{CuK} \alpha$ radiation (BrukerD8, $\lambda=0.15405 \mathrm{~nm})$. The absorption spectral characterization was performed using a Shimadzu UV-2550 spectrophotometer. Photoluminescence measurement was carried out employing a CARY Eclipse Fluorescence spectrum.

\section{Results and Discussion}

Figure 1(a)-(h) show the FE-SEM images of the $\mathrm{ZnO}$ nanorods arrays on zinc powder substrate with different reaction times. As shown in Figure 1(a), the zinc powders before reaction are

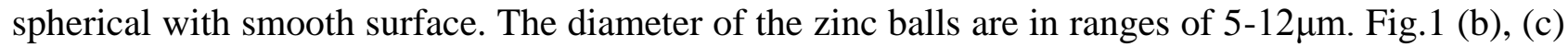
shows SEM image of the $\mathrm{ZnO}$ nanorods successfully synthesized using $\mathrm{Zn}$ powder as precursor at $180^{\circ} \mathrm{C}$ for $2 \mathrm{~h}$. It is clearly seen that $\mathrm{ZnO}$ nanorods in a hexagonal shape with the pencil shape tip have been grown on the surface of zinc balls. Most grown nanorods do not have a uniform diameter. In addition, we found after chemical etching on the surface of zinc microspheres become very rough. After a longer reaction time of $24 \mathrm{~h}$, high density of $\mathrm{ZnO}$ "dandelions" comprise numerous one-dimensional nanorods grow vertically from the substrates (Fig.1(d)-(g)). The diameter of the nanorods with the pencil shape tip is estimated to be $150-500 \mathrm{~nm}$. The length of the nanorods is more than $2 \mu \mathrm{m}$. The $\mathrm{ZnO}$ nanorods with their -c-axes ([0001]) pointing toward the center of dandelion sphere, and a large central space can be created (see a cracked opening, Fig.1(h)). In this way, $\mathrm{ZnO}$ dandelions with interior space had been achieved.

The XRD pattern of $\mathrm{ZnO}$ nanorods dandelions prepared at $180^{\circ} \mathrm{C}$ for $24 \mathrm{~h}$ are shown in Fig.2. The diffraction peaks are well indexed to the standard diffraction pattern of hexagonal phase $\mathrm{ZnO}$ (JCPDS 36-1451) with unit cell constants of $a=0.3250 \mathrm{~nm}, c=0.5207 \mathrm{~nm}$, implying a wurtzite structure with high crystallinity. The small diffraction peak of the metallic $\mathrm{Zn}$ which was not reacted completely can also be seen in the Fig.2.

Figure 3 depicts the schematic illustration of formation of $\mathrm{ZnO}$ dandelions microspheres. Firstly, an oxide layer is formed on the surface of the metallic $\mathrm{Zn}$ microspheres. With increasing reaction time, $\mathrm{Zn}$ atoms diffuse at liquid-solid interface and then turn into soluble zincate ions $\mathrm{ZnO}_{2}{ }^{2-}$. At the same time, $\mathrm{H}_{2}$ is released, which provides an additional driving force for $\mathrm{Zn}$ out-diffusion from the metal cores. The anions $\mathrm{ZnO}_{2}{ }^{2-}$ react with water and deposit back to the solid phase $\mathrm{ZnO}{ }^{[12]}$. With the continuous evacuation of $\mathrm{Zn}$ core, high density of $\mathrm{ZnO}$ nanorods grows nearly vertically from the $\mathrm{Zn}$ substrates. After the Kirkendall process, micrometer scale hollow $\mathrm{ZnO}$ dandelions organized by $\mathrm{ZnO}$ nanorods were formed. 


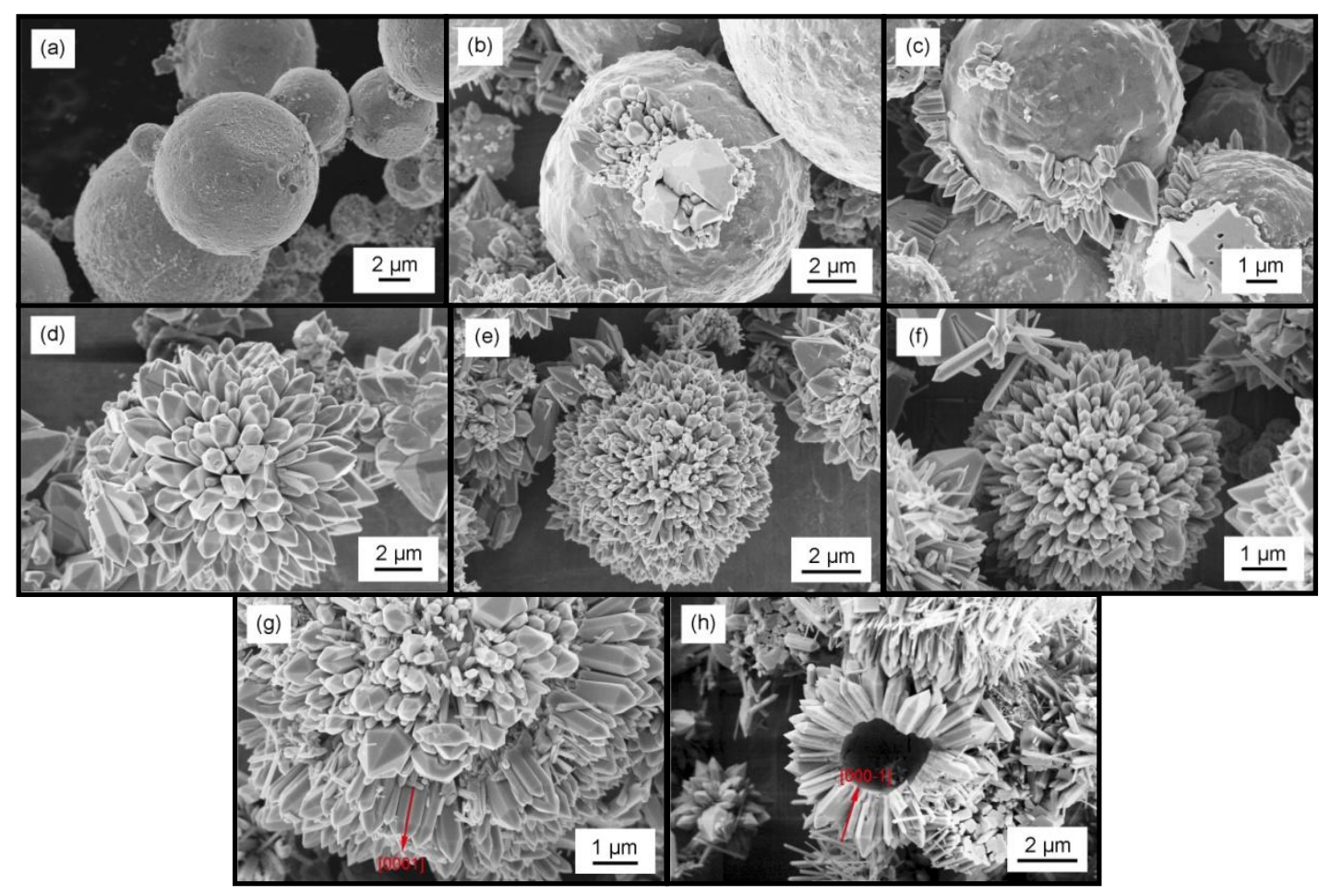

Fig.1 FE-SEM Images of ZnO Dandelions Prepared from Zn Microspheres for Different Hours: (a) The Zinc Powders before Reaction; (b), (c) $180^{\circ} \mathrm{C}$ for $2 \mathrm{~h}$; (d)-(h) $180^{\circ} \mathrm{C}$ for $24 \mathrm{~h}$

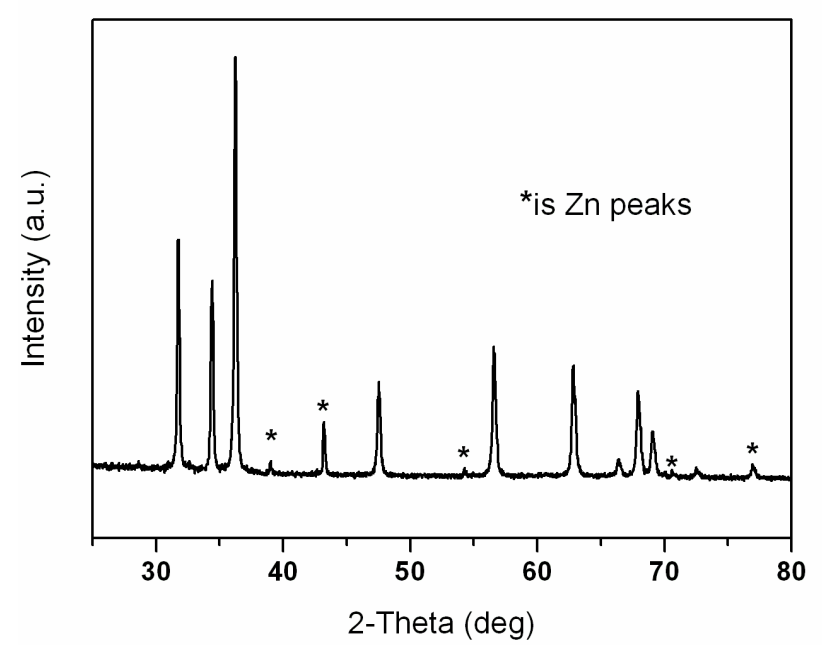

Fig. 2 XRD Patterns of the $\mathrm{ZnO}$ Dandelions Prepared at $180^{\circ} \mathrm{C}$ for $24 \mathrm{~h}$
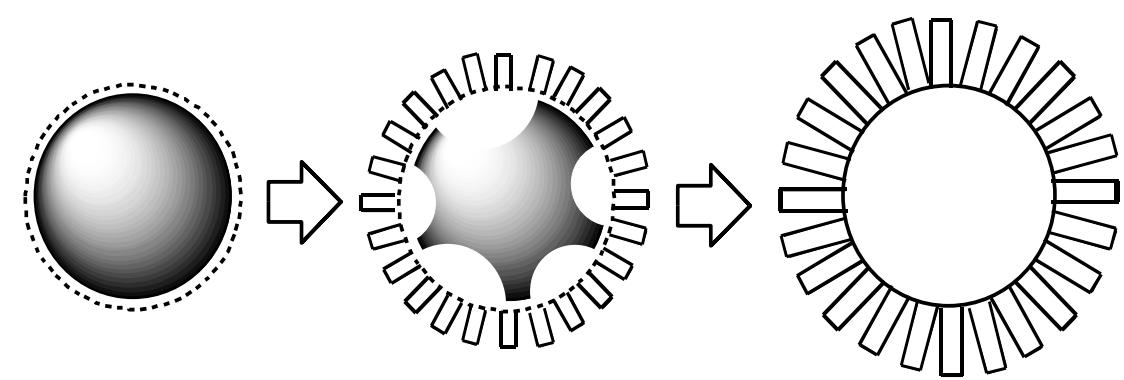

Fig.3 Schematic Illustration of Formation of $\mathrm{ZnO}$ Dandelions Microspheres 

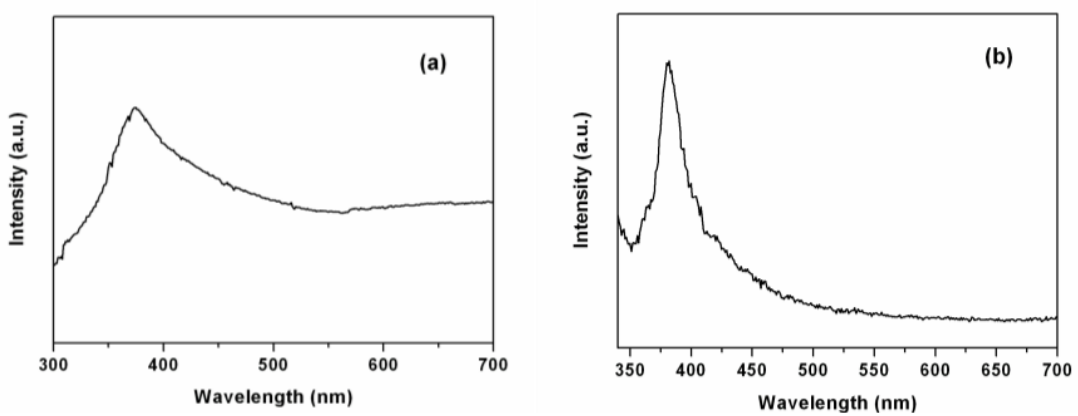

Fig.4 The Room-temperature Optical Absorption (a) and Photoluminescence Spectrum (b) of ZnO dandelions Microspheres

Fig.4(a) presents the room-temperature optical absorption of the $\mathrm{ZnO}$ "dandelions" by dispersing the sample in spectroscopic grade ethanol. The spectrum shown in the figure clearly indicates the excitionic character at room temperature. Generally an excitonic absorption peak appears if the defect density is considerably low. From the optical absorption spectrum it is evident that the $\mathrm{ZnO}$ nanorods so formed were of high optical quality.

Room temperature photoluminescence (PL) nolinebreak measurement of the as-grown $\mathrm{ZnO}$ nanorods was carried out by using a Xe lamp (325nm wavelength) as the source of excitation. Figure 4(b) shows the typical PL spectrum of the $\mathrm{ZnO}$ nanorods with a strong luminescence peak centered around $380 \mathrm{~nm}$. The UV emission corresponding to the near band edge emission of $\mathrm{ZnO}$ is attributed to the recombination of free excitons ${ }^{[13]}$. The strong room temperature UV emission property should be attributed to the high purity, good crystallinity of the as synthesized $\mathrm{ZnO}$ microspheres. This kind of hollow spherical $\mathrm{ZnO}$ structure with strong UV emission could be used in UV nano/micro-optoemission devices.

\section{Conclusion}

In summary, micrometer scale hollow $\mathrm{ZnO}$ dandelions organized by $\mathrm{ZnO}$ nanorods were formed via hydrothermal method, following a Kirkendall process. The room-temperature photoluminescence study reveals that the nanorods are of high purity and excellent optical quality, exhibiting very strong UV emission at $380 \mathrm{~nm}$. In principle, a lot of metal oxides and metal-semiconductor composites can be obtained in this synthetic architecture. Hopefully, they can be applied in many fields, such as light-generated electrons, three-dimensional lasing, and new photocatalysts.

\section{References}

[1] E. Kirkendall, L.Thomassen, C. Upthegrove, Mining, Metallurgical, and Petroleum Engineers, 133(1939) 186-203.

[2] A. Smigelskas, E. Kirkendall,Zinc diffusion in alpha brass, Transaction of American Institute of Mining, Metallurgical, and Petroleum Engineers.171(1947) 130-142.

[3] Y. D. Yin, R. M. Rioux, C. K. Erdonmez, et al., Formation of hollow nanocrystals through the nanoscalekirkendall effect, Science. 304 (2004) 711-714.

[4] Z.Y. Jiang, Z. X. Xie, X. H. Zhang, et al., Synthesis of single-crystalline ZnO polyhedral submicrometer-sized hollow beads using laser-Assisted growth with ethanol droplets as soft templates, Advanced Materials.16 (2004) 904-907.

[5] H. L. Xu, W. Z. Wang, Template synthesis of multishelled $\mathrm{Cu}_{2} \mathrm{O}$ hollow spheres with a single-crystalline shell wall, AngewandteChemie International Edition, 46 (2007)1489 1492.

[6] X. X. Li, Y. J. Xiong, Z. Q. Li, et al., Large-scale fabrication of $\mathrm{TiO}_{2}$ hierarchical hollow spheres, 
Inorganic Chemistry. 45 (2006) 3493-3495.

[7] Z. Zhong, Y. Yin, B. Gates, et al., Preparation of mesoscale hollow spheres of $\mathrm{TiO}_{2}$ and $\mathrm{SnO}_{2}$ by templating against crystalline arrays of polystyrene beads, Advanced Materials. 12 (2000) 206-209.

[8] M. H. Huang, S. Mao, H. Feick, et al., Room-temperature ultraviolet nanowire nanolasers, Science.292 (2001) 1897-1899.

[9] S. C. Minne, S. R. Manalis, C. F. Quate, Parallel atomic force microscopy using cantilevers with integrated piezoresistive sensors and integrated piezoelectric actutors, Applied Physics Letters. 67 (1995) 3918-3920.

[10] Q. Wan, Q. H. Li, Y. J. Chen, et al., Fabrication and ethanol sensing characteristics of ZnO nanowire gas sensors, Applied Physics Letters. 84 (2004) 3654-3656.

[11] Y. Tian, H. B. Lu, L. Liao, et al., Synthesis and evolution of hollow $\mathrm{ZnO}$ microspheres assisted by Zn powder precursor, Solid State Communications. 149 (2009) 456-460.

[12] B. Liu, H. C. Zeng, Fabrication of $\mathrm{ZnO}$ "Dandelions" via a modified Kirkendall process. Journal of the American Chemical Society, 126 (2004) 16744-16746.

[13] K. Vanheusden, W. L. Warren, C. H. Seager, et al., Mechanisms behind green photoluminescence in ZnO phosphor powders, Journal of Applied Physics. 79(1996)7983-7990. 